\title{
The Relationship between C-Reactive Protein/Albumin Ratio and Disease Activity in Patients with Inflammatory Bowel Disease
}

\author{
Yi-Han Chen $(\mathbb{D}$, Li Wang, Shu-Yi Feng, Wei-Min Cai, Xiao-Fu Chen, and Zhi-Ming Huang \\ Department of Gastroenterology and Hepatology, The First Affiliated Hospital of Wenzhou Medical University, \\ Wenzhou 325000, China \\ Correspondence should be addressed to Zhi-Ming Huang; wyyhzm@126.com
}

Received 25 February 2020; Revised 27 May 2020; Accepted 1 June 2020; Published 22 June 2020

Academic Editor: Walter Fries

Copyright ( 2020 Yi-Han Chen et al. This is an open access article distributed under the Creative Commons Attribution License, which permits unrestricted use, distribution, and reproduction in any medium, provided the original work is properly cited.

\begin{abstract}
Objectives. The aims of this study were to evaluate the C-reactive protein/albumin ratio (CRP/ALB), inflammatory markers, and parameters from the complete blood count (CBC) in patients with inflammatory bowel disease (IBD) and their associations with disease activity. Methods. A total of 876 IBD patients, composed of 275 patients with ulcerative colitis (UC) and 601 patients with Crohn's disease (CD), were included in this retrospective study, and the serum C-reactive protein (CRP), albumin (ALB), erythrocyte sedimentation rate (ESR), and CBC parameters were measured. To explore the disease activity, the Mayo score and Crohn disease activity index were used to assess UC and CD patients, respectively. Results. The CRP/ALB ratio, CRP, ESR, platelet to lymphocyte ratio (PLR), red blood cell distribution width (RDW), and neutrophil to lymphocyte ratio (NLR) levels in active IBD patients were significantly higher than those in inactive IBD patients, whereas ALB and lymphocyte to monocyte ratio (LMR) levels were significantly decreased $(P<0.001)$. The receiver operating characteristic analysis showed that the optimum cut-off values of the CRP/ALB ratio for active UC and CD were 0.18 and 0.43 , with sensitivities of $67.8 \%$ and $75.8 \%$ and specificities of $86.7 \%$ and $92.0 \%$, respectively. Multivariable logistic analysis revealed that after adjusting for these inflammatory markers (ESR, NLR, PLR, and LMR), the CRP/ALB ratio was a statistically significant parameter capable of differentiating the disease activity of UC and CD. Conclusions. This study indicated that the CRP/ALB ratio was closely related to the IBD disease activity. Compared with CBC parameters, the CRP/ALB ratio had a higher discriminative capacity for active IBD.
\end{abstract}

\section{Introduction}

Inflammatory bowel disease (IBD), a life-long disease resulting from the interaction of environmental and genetic elements, has been a global healthcare problem with a steadily increasing incidence [1]. IBD is mainly composed of two different bowel-relapsing disorders, including Crohn's disease (CD) and ulcerative colitis (UC). Early detection of the disease activity of IBD is of great significance for the treatment of this disease, which can effectively prevent complications and therefore improve prognosis as well as quality of life $[2,3]$. Biomarkers of IBD can provide helpful information regarding the disease activity. Despite many efforts in the discovery of new biomarkers [4-7], endoscopy continues to be the gold standard for the diagnosis and evaluation of the disease activity in IBD patients. Thus, further stud- ies are needed to find a cost-effective and noninvasive biomarker for clinical practice.

In current clinical practice, commonly used noninvasive biomarkers such as C-reactive protein (CRP) and erythrocyte sedimentation rate (ESR), are considered to be important for both early diagnosis and accurate monitoring of the disease activity in IBD patients [8]. As an acute phase protein, CRP measurements are widely available and relatively inexpensive to obtain; however, elevated serum CRP levels can also be affected by other extraintestinal inflammatory processes, and there is heterogeneity in the CRP production of individuals because of genetic differences [9]. ESR testing is less widely used than CRP testing due to the slower response to disease activity changes [10]. Fecal calprotectin has relatively better sensitivity and specificity and is the best clinical biological indicator for the assessment of the disease activity in IBD 
patients [11]; however, it is still not commonly used in clinical practice for multiple reasons including high cost, long time requirement, and the need to collect samples on demand. In the last few years, some systemic inflammatory markers obtained from the complete blood count (CBC), such as the neutrophil to lymphocyte ratio (NLR), platelet to lymphocyte ratio (PLR), and lymphocyte to monocyte ratio (LMR), have been reported as diagnostic and predictive indicators of IBD $[5,12,13]$. Moreover, a study showed that the red blood cell distribution width (RDW) can assess the disease activity of CD, but it does not perform well for UC [14]. Serum albumin (ALB) is generally recognized as an indicator of nutrition, and low ALB levels often indicate malnutrition. The rate of ALB synthesis is also directly affected by the severity of acute infection, given that ALB is a negative acute phase protein [15]. Furthermore, a recent study showed that ALB may have a diagnostic value in identifying children with potential CD [16].

The C-reactive protein/albumin ratio (CRP/ALB), determined by dividing the CRP level by the ALB level, was initially used as a novel predictor to identify critically ill patients in an acute medical ward [17]. In recent years, an increasing number of studies have suggested that an elevated $\mathrm{CRP} / \mathrm{ALB}$ ratio predicts a worse prognosis in cancer patients [18]. Moreover, the CRP/ALB ratio has also been considered a useful biomarker for evaluating the disease activity in patients with Takayasu arteritis [19]. However, there have been few previous studies on the CRP/ALB ratio in IBD patients, and one study showed the association between the CRP/ALB ratio and the disease activity of CD [20].

Therefore, in this study, we evaluated the CRP/ALB ratio, inflammatory markers, and CBC parameters in patients with IBD and their associations with the disease activity.

\section{Materials and Methods}

2.1. Participants. From September 2011 to September 2019, a total of $876 \mathrm{IBD}$ (275 UC, $601 \mathrm{CD}$ ) patients followed at the First Affiliated Hospital of Wenzhou Medical University were included in the current retrospective study. The study was approved by the hospital ethics committee. These patients were diagnosed with IBD based on standard clinical, laboratory, radiological, endoscopic, and histopathologic findings. Among $1511 \mathrm{IBD}$ patients who were retrospectively analyzed, only 876 subjects met the criteria for inclusion in this study. Patients who met the following criteria were excluded: other autoimmune diseases, acute or chronic renal failure, heart diseases, cirrhosis, cancer, acute or chronic infections, and missing ALB or CRP data at admission. We extracted patient information, including age, sex, smoking history, body mass index (BMI), disease duration, endoscopic findings, localization of the disease, and detailed medication list (corticosteroids, 5-aminosalicylate (5-ASA), immunosuppressant, and biologics), from the medical records. The serum CRP level, ESR, ALB, and CBC parameters were also measured in all patients. The following ratios of inflammatory markers were calculated: CRP/ALB ratio, NLR, LMR, and PLR.
2.2. Disease Activity. The disease activity of UC and CD patients was evaluated by the Mayo score and Crohn disease activity index (CDAI), respectively. The Mayo score, a composite score incorporating 4 items: stool frequency, rectal bleeding, findings of flexible proctosigmoidoscopy, and physician's global assessment, was introduced more than 30 years ago and allows a simple and effective classification of UC patients [21, 22]. UC patients whose Mayo scores were $>2$ were divided into the active UC group. CD patients were categorized using the CDAI criteria, which includes weight, general health status, number of daily bloody stools, degree of abdominal pain, hematocrit, and complications [23]. CD patients with CDAI $>150$ were divided into the active CD group.

2.3. Statistical Analysis. The Statistical Package for Social Sciences (SPSS) 22.0 for Windows was used for the statistical analyses. The normality of the distribution of continuous variables was determined by the Kolmogorov-Smirnov test. Continuous values were presented as the mean \pm standard deviation or, in case of nonnormally distributed data, as the median and 25th-75th percentiles. The chi-square test was used to analyze categorical variables. Comparisons of normally distributed continuous variables were carried out using independent sample $t$-tests or paired $t$-tests. The Mann-Whitney $U$ test for independent subgroups and the Wilcoxon test for dependent subgroups were used for the analysis of nonnormally distributed data. To determine the relationships of the CRP/ALB ratio and other laboratory parameters with the IBD activity, Spearman's correlation analysis was used. Multivariate logistic regression for the CRP/ALB ratio, ESR, NLR, PLR, and LMR was carried out to reveal which biomarkers were significantly associated with the disease activity in UC and CD patients. To differentiate between active and inactive patients with IBD, the optimal cut-off values of the CRP/ALB ratio, ALB, CRP, ESR, NLR, PLR, and LMR with maximum sensitivity and specificity were calculated via receiver operating characteristic (ROC) curve analysis. The $Z$-statistic was used to compare the areas under the ROC curves (AUCs). $P<0.05$ was considered to be statistically significant.

\section{Results}

The total 876 IBD patients included in the study consisted of 275 UC patients and $601 \mathrm{CD}$ patients. Among UC and CD patients, the median age was 48 and 27 years, respectively, with men accounting for $54.9 \%$ and $72.9 \%$, respectively. Based on the criteria described above, 275 UC patients were divided into "UC remission" (98) and "UC active" (177), and $601 \mathrm{CD}$ patients were divided into "CD remission" (299) and "CD active" (302). The demographic and clinical characteristics of subjects with UC and CD were shown in Table 1 .

No significant differences in age or sex were observed between the active and inactive groups, in either the UC or CD patients $(P>0.05)$. Table 2 shows that the CRP/ALB ratio, ESR, CRP, RDW, NLR, and PLR levels in the active $\mathrm{UC}$ group were significantly higher than those in the inactive 
TABLE 1: Characteristics of the patients in the UC and CD cohorts.

\begin{tabular}{lcc}
\hline & UC $(n=275)$ & CD $(n=601)$ \\
\hline Age (years) & $48(36-61)$ & $27(22-33)$ \\
Sex $(\mathrm{F} / \mathrm{M})$ & $124 / 151$ & $163 / 438$ \\
Smoking (yes/no) & $47 / 228$ & $90 / 511$ \\
BMI $\left(\mathrm{kg} / \mathrm{m}^{2}\right)$ & $20.06(18.44-21.28)$ & $19.33(17.44-20.9)$ \\
Duration of disease & $15.83(6-48)$ & $22.07(7.3-43.88)$ \\
(months) & $98(35.6 \%)$ & $299(49.8 \%)$ \\
Remission & $177(64.4 \%)$ & $302(50.2 \%)$ \\
Active disease & & - \\
Localization of disease & $49(17.8 \%)$ & - \\
$\quad$ Proctitis & $122(44.4 \%)$ & - \\
Left-side colitis & $104(37.8 \%)$ & $139(23.1 \%)$ \\
Extensive colitis & - & $117(19.5 \%)$ \\
Terminal ileitis & - & $345(57.4 \%)$ \\
Colitis & - & $267(44.4 \%)$ \\
$\quad$ Ileocolitis & $102(37.1 \%)$ & $468(77.9 \%)$ \\
Medication history & $234(85.1 \%)$ & $283(47.1 \%)$ \\
Steroids & $31(11.3 \%)$ & $(56.1 \%)$ \\
5-ASA & $22(8.0 \%)$ & \\
Immunosuppressant & & \\
Biologics & &
\end{tabular}

Values are expressed as $n$ (\%) or median (IQR). UC: ulcerative colitis; CD: Crohn's disease; BMI: body mass index.

UC group, whereas the ALB and LMR levels were significantly lower $(P<0.001)$. With respect to CD patients, BMI, CRP/albumin ratio, ESR, CRP, RDW, NLR, PLR, LMR, and ALB levels were also significantly different between patients with active disease and patients in remission $(P<0.001)$, as shown in Table 3.

As shown in Table 4, the results of the Spearman correlation analysis showed correlations between laboratory parameters and the IBD activity. ESR $(r=0.421, P<0.001)$, CRP $(r=0.595, P<0.001)$, and the CRP/ALB ratio $(r=0.637$, $P<0.001)$ had significant correlations with the UC activity, while ALB $(r=-0.692, P<0.001)$ and LMR $(r=-0.495$, $P<0.001)$ were negatively associated with UC disease activity. In addition, the Spearman correlation analysis in $\mathrm{CD}$ patients also indicated significant positive correlations of the CD activity with ESR $(r=0.63, P<0.001)$, CRP $(r=0.731, P<0.001)$, and the CRP/ALB ratio $(r=0.763$, $P<0.001)$ and negative correlations with $\operatorname{ALB}(r=-0.748$, $P<0.001)$ and LMR $(r=-0.454, P<0.001)$.

The results of multivariate logistic regression analysis for exploring the associations of the CRP/ALB ratio, ESR, NLR, PLR, and LMR with active UC and CD were shown in Table 5. After adjusting for these inflammatory markers (ESR, NLR, PLR, and LMR), the odds ratios of the CRP/ALB ratio in UC and CD patients were 1.359 (95\% confidence interval, 1.144-1.615) and 1.569 (95\% confidence interval, 1.413-1.741), respectively. Therefore, the CRP/ALB ratio was an independent predictive factor of the disease activity of UC and CD.
The predictive values of ESR, CRP, CRP/ALB ratio, albumin, NLR, PLR, and LMR for differentiating active from inactive $\mathrm{UC}$ and $\mathrm{CD}$ were investigated by the receiver operating characteristic (ROC) curve analysis. The results of this analysis are shown in Tables 6 and 7. As shown in Figures 1 and 2, the discriminatory values of these indicators relative to the UC and $\mathrm{CD}$ activities were depicted. The results of ROC analysis showed that the AUC of ALB in UC patients was 0.845 , which was higher than that of CRP $(0.806)$ and the CRP/ALB ratio (0.827) $(P>0.05)$. However, we found that the AUC of the CRP/ALB ratio (0.925) was higher than that of CRP (0.91) and ALB (0.893) in CD patients $(P>0.05)$. It is worth noting that according to the $Z$-statistic, the AUCs of the NLR, PLR, LMR, and ESR were all lower than that of the CRP/ALB ratio in both UC and CD patients $(P<0.05)$.

\section{Discussion}

The aim of this retrospective study was to explore the use of the CRP/ALB ratio as a new noninvasive biomarker for evaluating the disease activity of IBD. In our study, significant increases in the CRP/ALB ratio, ESR, RDW, NLR, PLR, and CRP levels and significant decreases in LMR and ALB levels were observed in the active groups compared to the respective levels in the remitted IBD group. Furthermore, these parameters correlated strongly with UC and CD activities. In addition, we also found that among IBD patients, the BMI of active patients was lower than that of patients in remission $(P<0.05)$. This means that the nutritional status of patients in the active phase is worse, indicating that the nutritional status of patients is related to the disease activity. ROC analysis suggested that the AUC of ALB in UC patients was 0.845 , which was higher than that of CRP $(0.806)$ and the CRP/ALB ratio $(0.827)(P>0.05)$, but for patients with $C D$, the AUC of the CRP/ALB ratio (0.925) was higher than the AUCs of CRP (0.91) and ALB (0.893; $P>0.05)$. After adjusting for the other inflammatory markers (NLR, ESR, PLR, and LMR), the CRP/ALB ratio was an independent predictive factor for the disease activity of UC and CD.

The analysis of components of the $\mathrm{CBC}$ is a simple and inexpensive method to assess the disease activity of IBD. The association between $\mathrm{CBC}$ parameters and the disease activity in UC and CD patients has previously been studied $[5,6,14]$. It has previously been shown that in patients with active UC and CD, the RDW, the NLR, and the PLR were increased, and the LMR was decreased. These previous findings support the results of the present study. However, as previous studies reported that the NLR had no value for discriminating the disease activity, the role of the NLR in predicting the UC and CD disease activities remains controversial $[24,25]$. Xu et al. [26] reported that a low LMR might be an effective biomarker for identifying the disease activity of UC, but this is not the case when evaluating the disease activity of CD. In our study, according to the results of multivariate logistic regression analysis, we found that LMR has a good value in differentiating the disease activity of UC, which is basically close to the findings of previous studies. Moreover, we found that the NLR was well 
TABLE 2: Differences between active disease and remission in UC patients.

\begin{tabular}{|c|c|c|c|}
\hline Parameters & UC active $(n=177)$ & UC remission $(n=98)$ & $P$ value \\
\hline Age (years) & $49(37-61)$ & $46.5(33.25-61)$ & 0.323 \\
\hline $\operatorname{Sex}(F / M)$ & $76 / 101$ & $48 / 50$ & 0.335 \\
\hline Smoking (yes/no) & $30 / 147$ & $17 / 81$ & 0.933 \\
\hline BMI $\left(\mathrm{kg} / \mathrm{m}^{2}\right)$ & $19.27(18.27-20.81)$ & $20.64(18.83-21.89)$ & $<0.001$ \\
\hline $\operatorname{ESR}(\mathrm{mm} / \mathrm{h})$ & $22(10.5-37.5)$ & $6(2-18)$ & $<0.001$ \\
\hline CRP (mg/L) & $12.7(3.65-32.55)$ & $2.94(1.26-4.09)$ & $<0.001$ \\
\hline $\operatorname{ALB}(g / L)$ & $34.2 \pm 5.9$ & $41.7 \pm 4.5$ & $<0.001$ \\
\hline CRP/ALB & $0.36(0.1-0.97)$ & $0.06(0.03-0.1)$ & $<0.001$ \\
\hline Neutrophil $\left(10^{9} / \mathrm{L}\right)$ & $4.6(3.42-7.03)$ & $3.55(2.7-4.93)$ & $<0.001$ \\
\hline Monocyte $\left(10^{9} / \mathrm{L}\right)$ & $0.7(0.46-0.92)$ & $0.48(0.38-0.63)$ & $<0.001$ \\
\hline Lymphocyte $\left(10^{9} / \mathrm{L}\right)$ & $1.76(1.33-2.23)$ & $1.91(1.5-2.49)$ & 0.063 \\
\hline $\operatorname{Hgb}(\mathrm{g} / \mathrm{L})$ & $119(103.5-132)$ & $131(120.75-139)$ & $<0.001$ \\
\hline $\operatorname{MCV}(\mathrm{fl})$ & $88.9(82.85-92.75)$ & $89(86.57-93.1)$ & 0.035 \\
\hline RDW (\%) & $14(13.45-15.05)$ & $13.3(12.7-14.3)$ & $<0.001$ \\
\hline Platelet $\left(10^{9} / \mathrm{L}\right)$ & $281(210-374)$ & $236(199.25-284)$ & $<0.001$ \\
\hline MPV (fl) & $9.9(9.3-11)$ & $10.3(9.6-11.23)$ & 0.074 \\
\hline NLR & $2.82(1.77-4.21)$ & $1.84(1.32-2.81)$ & $<0.001$ \\
\hline PLR & $163.38(121.28-222.53)$ & $122.74(92.31-168.64)$ & $<0.001$ \\
\hline LMR & $2.67(1.8-3.5)$ & $4.16(3.17-5.14)$ & $<0.001$ \\
\hline
\end{tabular}

Values are expressed as the mean \pm SD or median (IQR). UC: ulcerative colitis; BMI: body mass index; ESR: erythrocyte sedimentation rate; CRP: C-reactive protein; ALB: albumin; MCV: mean corpuscular volume; RDW: red cell distribution width; MPV: mean platelet volume; NLR: neutrophil to lymphocyte ratio; PLR: platelet to lymphocyte ratio; LMR: lymphocyte to monocyte ratio.

TABLE 3: Differences between active disease and remission in CD patients.

\begin{tabular}{|c|c|c|c|}
\hline Parameters & CD active $(n=302)$ & CD remission $(n=299)$ & $P$ value \\
\hline$\overline{\text { Age (years) }}$ & $26(22-35)$ & $27(22-31)$ & 0.910 \\
\hline $\operatorname{Sex}(F / M)$ & $81 / 221$ & $82 / 217$ & 0.868 \\
\hline Smoking (yes/no) & $51 / 251$ & $39 / 260$ & 0.187 \\
\hline BMI $\left(\mathrm{kg} / \mathrm{m}^{2}\right)$ & $18.16(16.55-20.15)$ & $19.56(18.25-21.6)$ & $<0.001$ \\
\hline $\operatorname{ESR}(\mathrm{mm} / \mathrm{h})$ & $30.5(17-47)$ & $7(2-14)$ & $<0.001$ \\
\hline CRP (mg/L) & $30(14.9-59.8)$ & $3.07(1.89-9.09)$ & $<0.001$ \\
\hline $\mathrm{ALB}(\mathrm{g} / \mathrm{L})$ & $33.3 \pm 5.0$ & $41.7 \pm 4.7$ & $<0.001$ \\
\hline CRP/ALB & $0.88(0.43-1.86)$ & $0.07(0.04-0.22)$ & $<0.001$ \\
\hline Neutrophil $\left(10^{9} / \mathrm{L}\right)$ & $5.01(3.48-7.07)$ & $3.46(2.61-4.23)$ & $<0.001$ \\
\hline Monocyte $\left(10^{9} / \mathrm{L}\right)$ & $0.69(0.47-0.9)$ & $0.51(0.39-0.63)$ & $<0.001$ \\
\hline Lymphocyte $\left(10^{9} / \mathrm{L}\right)$ & $1.25(0.9-1.67)$ & $1.57(1.14-1.95)$ & $<0.001$ \\
\hline $\operatorname{Hgb}(\mathrm{g} / \mathrm{L})$ & $115(101-127)$ & $135(123-149)$ & $<0.001$ \\
\hline MCV (fl) & $83.25(78.43-87.6)$ & $88.4(84.8-92.1)$ & $<0.001$ \\
\hline RDW (\%) & $14.8(13.3-16.53)$ & $13.3(12.7-14.7)$ & $<0.001$ \\
\hline Platelet $\left(10^{9} / \mathrm{L}\right)$ & $327.5(253.75-410.5)$ & $246(206-294)$ & $<0.001$ \\
\hline MPV (fl) & $9.6(8.9-10.4)$ & $10.6(10-11.4)$ & $<0.001$ \\
\hline NLR & $4.08(2.78-6.13)$ & $2.17(1.52-3.31)$ & $<0.001$ \\
\hline PLR & $264.84(192.08-367.64)$ & $165.61(118.72-230)$ & $<0.001$ \\
\hline LMR & $1.89(1.36-2.57)$ & $3.05(2.27-4.25)$ & $<0.001$ \\
\hline
\end{tabular}

Values are expressed as the mean \pm SD or median (IQR). CD: Crohn's disease; BMI: body mass index; ESR: erythrocyte sedimentation rate; CRP: C-reactive protein; ALB: albumin; MCV: mean corpuscular volume; RDW: red cell distribution width; MPV: mean platelet volume; NLR: neutrophil to lymphocyte ratio; PLR: platelet to lymphocyte ratio; LMR: lymphocyte to monocyte ratio. 
TABLE 4: Spearman correlation coefficients between laboratory parameters and the disease activity of UC and CD.

\begin{tabular}{|c|c|c|c|c|}
\hline \multirow{2}{*}{ Parameters } & \multicolumn{2}{|c|}{ Mayo UC score } & \multicolumn{2}{|c|}{ CDAI } \\
\hline & $r$ value & $P$ value & $r$ value & $P$ value \\
\hline ESR & 0.421 & $<0.001$ & 0.630 & $<0.001$ \\
\hline CRP & 0.595 & $<0.001$ & 0.731 & $<0.001$ \\
\hline ALB & -0.692 & $<0.001$ & -0.748 & $<0.001$ \\
\hline CRP/ALB & 0.637 & $<0.001$ & 0.763 & $<0.001$ \\
\hline Neutrophil & 0.334 & $<0.001$ & 0.370 & $<0.001$ \\
\hline Monocyte & 0.354 & $<0.001$ & 0.270 & $<0.001$ \\
\hline Lymphocyte & -0.203 & 0.001 & -0.241 & $<0.001$ \\
\hline $\mathrm{Hgb}$ & -0.402 & $<0.001$ & -0.598 & $<0.001$ \\
\hline $\mathrm{MCV}$ & -0.212 & $<0.001$ & -0.389 & $<0.001$ \\
\hline RDW & 0.341 & $<0.001$ & 0.370 & $<0.001$ \\
\hline Platelet & 0.243 & $<0.001$ & 0.369 & $<0.001$ \\
\hline MPV & -0.144 & 0.017 & -0.449 & $<0.001$ \\
\hline NLR & 0.393 & $<0.001$ & 0.451 & $<0.001$ \\
\hline PLR & 0.350 & $<0.001$ & 0.442 & $<0.001$ \\
\hline LMR & -0.495 & $<0.001$ & -0.454 & $<0.001$ \\
\hline
\end{tabular}

UC: ulcerative colitis; CD: Crohn's disease; CDAI: Crohn's disease activity index; ESR: erythrocyte sedimentation rate; CRP: C-reactive protein; ALB: albumin; MCV: mean corpuscular volume; RDW: red cell distribution width; MPV: mean platelet volume; NLR: neutrophil to lymphocyte ratio; PLR: platelet to lymphocyte ratio; LMR: lymphocyte to monocyte ratio.

TABLE 5: Results of multivariate logistic regression analysis in patients with UC and CD.

\begin{tabular}{|c|c|c|c|c|c|}
\hline & Variables & $B$ & $P$ & Odds ratio & $95 \% \mathrm{CI}$ \\
\hline \multirow{5}{*}{ UC } & CRP/ALB & 0.307 & $<0.001$ & 1.359 & $1.144-1.615$ \\
\hline & ESR & 0.021 & 0.087 & 1.021 & 0.997-1.045 \\
\hline & NLR & -0.085 & 0.304 & 0.919 & $0.782-1.080$ \\
\hline & PLR & 0.004 & 0.156 & 1.004 & 0.999-1.009 \\
\hline & LMR & -0.247 & 0.002 & 0.781 & $0.666-0.915$ \\
\hline \multirow{5}{*}{$\mathrm{CD}$} & CRP/ALB & 0.450 & $<0.001$ & 1.569 & $1.413-1.741$ \\
\hline & ESR & 0.029 & 0.001 & 1.029 & $1.011-1.047$ \\
\hline & NLR & 0.160 & 0.026 & 1.174 & $1.019-1.351$ \\
\hline & PLR & 0.001 & 0.235 & 1.001 & 0.999-1.004 \\
\hline & LMR & -0.031 & 0.657 & 0.969 & $0.844-1.113$ \\
\hline
\end{tabular}

UC: ulcerative colitis; CD: Crohn's disease; ESR: erythrocyte sedimentation rate; NLR: neutrophil to lymphocyte ratio; PLR: platelet to lymphocyte ratio; LMR: lymphocyte to monocyte ratio; CI: confidence interval.

correlated with the activity of $\mathrm{CD}$, so there are still controversies about the NLR.

CRP and ALB, known as positive and negative acute phase reactants, respectively, are commonly used to assess inflammatory processes. Previous research also confirms that the inflammatory response can influence ALB synthesis [27]. The CRP/ALB ratio is considered a novel inflammationbased score that provides more useful information about inflammatory status than CRP or albumin alone in septic patients [28]. Qin et al. [20] found that the CRP/ALB ratio and ALB levels had a predictive value in determining the $\mathrm{CD}$ disease activity and were more significant in men. The retrospective study of 100 patients with active or inactive CD showed that the optimal cut-off point of the CRP/ALB ratio for active $C D$ was 0.69 , with a sensitivity and specificity of $59.7 \%$ and $81.6 \%$, respectively. In our study, we found that the optimal cut-off point of the CRP/ALB ratio for active CD was 0.43 , with a sensitivity of $75.8 \%$ and a specificity of $92 \%$, and the AUC of the CRP/ALB ratio was 0.925 , which was higher than that of $\operatorname{ALB}(0.893 ; P>0.05)$. The sensitivity and specificity of the CRP/ALB ratio in this study were higher than those in a previous study, which we consider to be mainly because the $\mathrm{CD}$ patients in the remission period we included had a milder status. In the present study, the CRP/ALB ratio showed a stronger correlation with the disease activity in IBD patients than the components of the $\mathrm{CBC}$. According to the results of the $Z$ statistic, the AUCs of the NLR, PLR, LMR, and ESR were all lower than that of the CRP/ALB ratio in both $\mathrm{UC}$ and $\mathrm{CD}$ patients $(P<0.05)$. In addition, the results 
TABLE 6: Accuracy of CRP/ALB and other inflammatory markers in differentiating active from inactive UC.

\begin{tabular}{|c|c|c|c|c|c|c|}
\hline Parameters & AUCs & SE & $95 \% \mathrm{CI}$ & Cut-offs & Sensitivity (\%) & Specificity (\%) \\
\hline CRP/ALB & 0.827 & 0.025 & $0.777-0.870$ & 0.18 & 67.8 & 86.7 \\
\hline CRP & 0.806 & 0.026 & $0.754-0.851$ & 5.38 & 80.6 & 70.1 \\
\hline ALB & 0.845 & 0.024 & $0.797-0.885$ & 38.20 & 73.5 & 80.6 \\
\hline ESR & 0.739 & 0.031 & $0.683-0.790$ & 15.00 & 67.8 & 72.5 \\
\hline NLR & 0.665 & 0.034 & $0.606-0.721$ & 2.40 & 57.1 & 70.4 \\
\hline PLR & 0.660 & 0.034 & $0.601-0.716$ & 187.68 & 42.4 & 86.7 \\
\hline LMR & 0.752 & 0.030 & $0.697-0.802$ & 3.56 & 76.8 & 69.4 \\
\hline
\end{tabular}

UC: ulcerative colitis; AUC: area under the curve; SE: standard error; CI: confidence interval; CRP: C-reactive protein; ALB: albumin; MCV: mean corpuscular volume; ESR: erythrocyte sedimentation rate; NLR: neutrophil to lymphocyte ratio; PLR: platelet to lymphocyte ratio; LMR: lymphocyte to monocyte ratio.

TABLE 7: Accuracy of CRP/ALB and other inflammatory markers in differentiating active from inactive CD.

\begin{tabular}{lcccccc}
\hline Parameters & AUCs & SE & $95 \%$ CI & Cut-offs & Sensitivity (\%) & Specificity (\%) \\
\hline CRP/ALB & 0.925 & 0.010 & $0.901-0.945$ & 0.43 & 75.8 & 76.2 \\
CRP & 0.910 & 0.011 & $0.884-0.932$ & 14.70 & 36.0 & 88.6 \\
ALB & 0.893 & 0.013 & $0.866-0.917$ & 20.90 & 69.9 & 83.3 \\
ESR & 0.851 & 0.016 & $0.820-0.878$ & 3.32 & 65.9 & 75.9 \\
NLR & 0.764 & 0.019 & $0.728-0.798$ & 191.22 & 76.2 & 60.9 \\
PLR & 0.747 & 0.020 & $0.710-0.781$ & 2.67 & 78.8 & 61.9 \\
LMR & 0.755 & 0.020 & $0.719-0.789$ &
\end{tabular}

CD: Crohn's disease; AUC: area under the curve; SE: standard error; CI: confidence interval; CRP: C-reactive protein; ALB: albumin; MCV: mean corpuscular volume; ESR: erythrocyte sedimentation rate; NLR: neutrophil to lymphocyte ratio; PLR: platelet to lymphocyte ratio; LMR: lymphocyte to monocyte ratio.

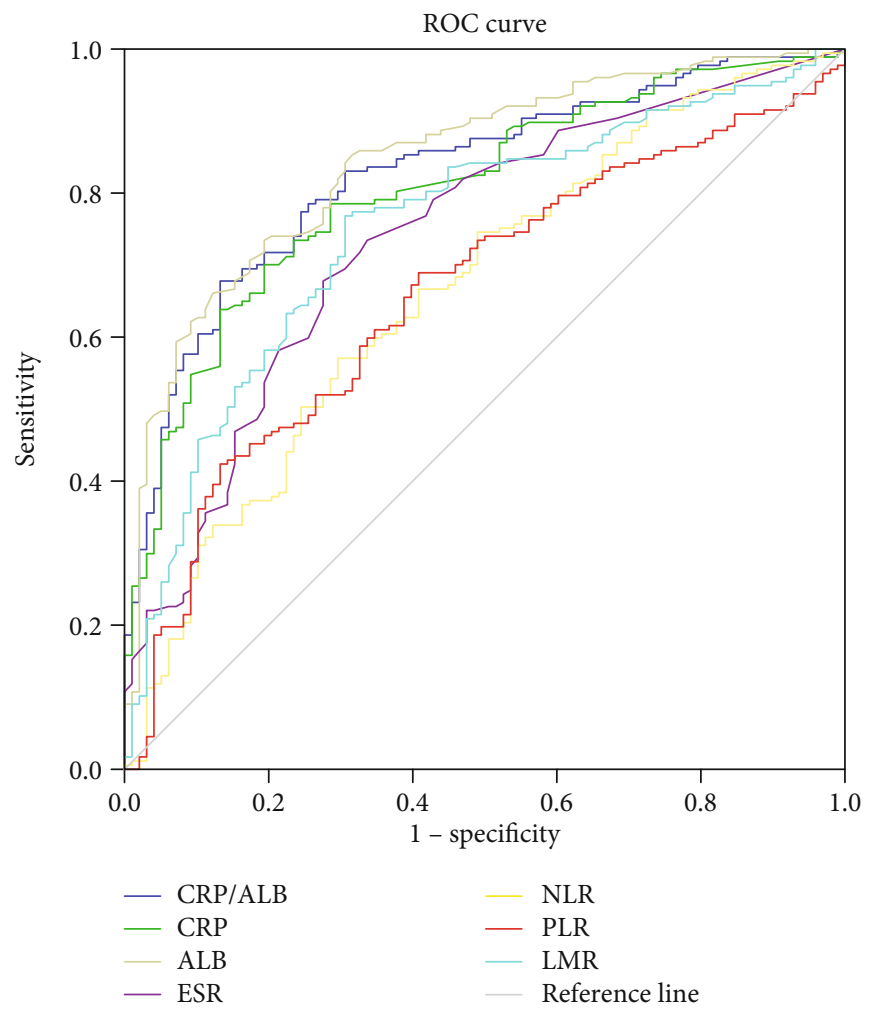

FIGURE 1: Receiver operating characteristic (ROC) curve of CRP/ALB vs. other inflammatory markers in predicting active UC. 


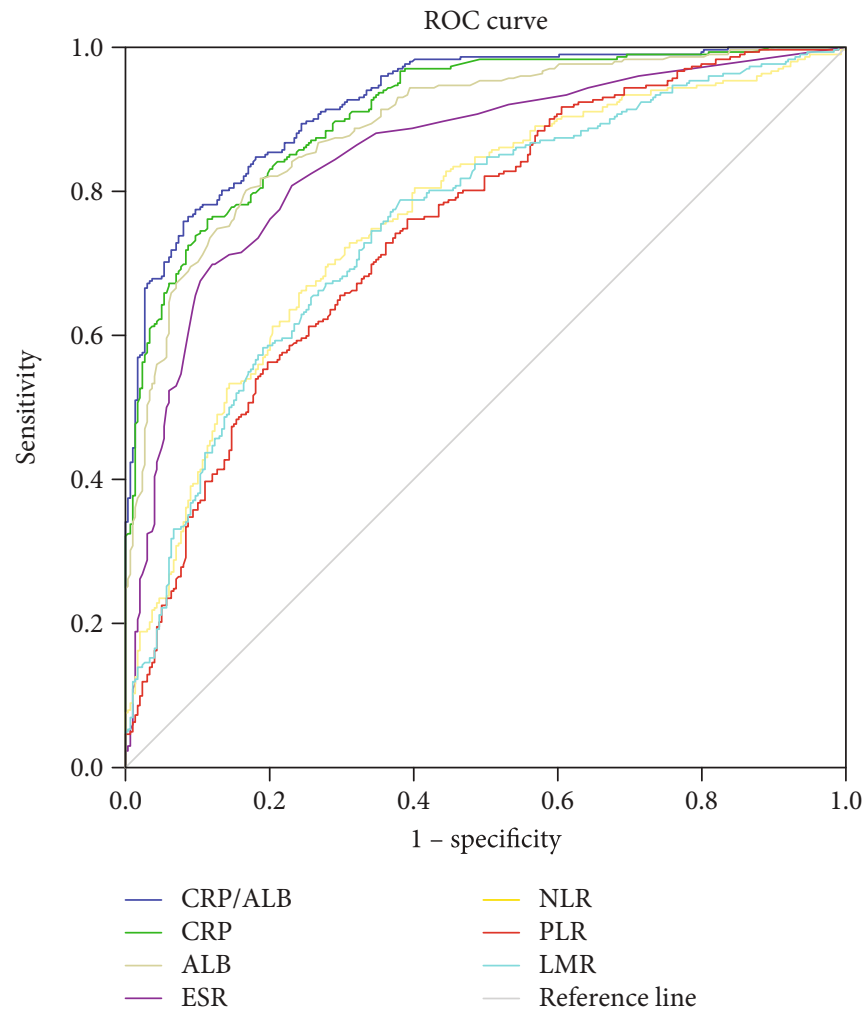

FIGURE 2: Receiver operating characteristic (ROC) curve of CRP/ALB vs. other inflammatory markers in predicting active CD.

of the multivariate analysis evaluating statistically significant parameters suggested that the CRP/ALB ratio was an important parameter capable of determining the disease activity of UC and CD.

Our study had some limitations. First, the patients we included were all followed at our hospital in southeastern China. Therefore, the results may not represent the general population in China. Second, our study was not designed to elucidate the mechanism that led to the observed increases of the CRP/ALB ratio in patients with active UC and CD. Third, this study did not evaluate other factors, such as the use of immunosuppressive agents and corticosteroids, which may affect the level of inflammatory markers. Finally, our study was designed as a retrospective single-center study, which prevents firm conclusions from being drawn. Prospective studies are needed to provide more useful information on this subject.

\section{Conclusions}

The findings from this study demonstrate that in subjects with IBD, the CRP/ALB ratio was strongly related to the disease activity. Compared with parameters from the $\mathrm{CBC}$, the CRP/ALB ratio had a higher discriminative capacity for active IBD. After adjusting for these inflammatory markers (ESR, NLR, PLR, and LMR), the CRP/ALB ratio was a helpful biomarker to differentiate the disease activity of UC and CD.

\section{Data Availability}

The data used to support the findings of this study are available from the corresponding author upon request.

\section{Conflicts of Interest}

All authors declare that they have no competing interests.

\section{References}

[1] N. A. Molodecky, I. S. Soon, D. M. Rabi et al., "Increasing incidence and prevalence of the inflammatory bowel diseases with time, based on systematic review," Gastroenterology, vol. 142, no. 1, pp. 46-54.e42, 2012, quiz e30.

[2] F. Magro, P. Gionchetti, R. Eliakim et al., "Third European evidence-based consensus on diagnosis and management of ulcerative colitis. Part 1: definitions, diagnosis, extra-intestinal manifestations, pregnancy, cancer surveillance, surgery, and ileo-anal pouch disorders," Journal of Crohn's \& Colitis, vol. 11, no. 6, pp. 649-670, 2017.

[3] F. Gomollón, A. Dignass, V. Annese et al., "3rd European evidence-based consensus on the diagnosis and management of Crohn's disease 2016: part 1: diagnosis and medical management," Journal of Crohn's \& Colitis, vol. 11, no. 1, pp. 3-25, 2016.

[4] W. A. Faubion Jr., J. G. Fletcher, S. O’Byrne et al., "EMerging BiomARKers in Inflammatory Bowel Disease (EMBARK) study identifies fecal calprotectin, serum MMP9, and serum IL-22 as a novel combination of biomarkers for Crohn's 
disease activity: role of cross-sectional imaging," The American Journal of Gastroenterology, vol. 108, no. 12, pp. 1891-1900, 2013.

[5] C. E. Cherfane, L. Gessel, D. Cirillo, M. B. Zimmerman, and S. Polyak, "Monocytosis and a low lymphocyte to monocyte ratio are effective biomarkers of ulcerative colitis disease activity," Inflammatory Bowel Diseases, vol. 21, no. 8, pp. 17691775, 2015.

[6] G. Acarturk, A. Acay, K. Demir, M. S. Ulu, A. Ahsen, and S. Yuksel, "Neutrophil-to-lymphocyte ratio in inflammatory bowel disease - as a new predictor of disease severity," Bratislavské Lekárske Listy, vol. 116, no. 4, pp. 213-217, 2015.

[7] C. S. Ferrer, M. A. Barno, E. M. Arranz et al., "The use of serum calprotectin as a biomarker for inflammatory activity in inflammatory bowel disease," Revista Española de Enfermedades Digestivas, vol. 111, no. 10, pp. 744-749, 2019.

[8] J. D. Lewis, "The utility of biomarkers in the diagnosis and therapy of inflammatory bowel disease," Gastroenterology, vol. 140, no. 6, pp. 1817-1826.e2, 2011.

[9] P. Henderson, N. A. Kennedy, J. E. van Limbergen et al., "Serum C-reactive protein and CRP genotype in pediatric inflammatory bowel disease: influence on phenotype, natural history, and response to therapy," Inflammatory Bowel Diseases, vol. 21, no. 3, pp. 596-605, 2015.

[10] E. A. Fagan, R. F. Dyck, P. N. Maton et al., "Serum levels of C-reactive protein in Crohn's disease and ulcerative colitis," European Journal of Clinical Investigation, vol. 12, no. 4, pp. 351-359, 1982.

[11] M. H. Mosli, G. Zou, S. K. Garg et al., "C-reactive protein, fecal calprotectin, and stool lactoferrin for detection of endoscopic activity in symptomatic inflammatory bowel disease patients: a systematic review and meta-analysis," The American Journal of Gastroenterology, vol. 110, no. 6, pp. 802-819, 2015, quiz 820.

[12] J. R. Feng, X. Qiu, F. Wang et al., "Diagnostic Value of Neutrophil-to-Lymphocyte Ratio and Platelet-toLymphocyte Ratio in Crohn's Disease," Gastroenterology Research and Practice, vol. 2017, Article ID 3526460, 5 pages, 2017.

[13] M. Y. Akpinar, Y. O. Ozin, M. Kaplan et al., "Platelet-to-lymphocyte ratio and neutrophil-to-lymphocyte ratio predict mucosal disease severity in ulcerative colitis," Journal of Medical Biochemistry, vol. 37, no. 2, pp. 155-162, 2018.

[14] A. Yeşil, E. Şenateş, İ. V. Bayoğlu, E. D. Erdem, R. . Demirtunç, and A. O. K. Övünç, "Red cell distribution width: a novel marker of activity in Infl ammatory bowel disease," Gut Liver, vol. 5, no. 4, pp. 460-467, 2011.

[15] B. R. Don and G. Kaysen, "Serum albumin: relationship to inflammation and nutrition," Seminars in Dialysis, vol. 17, no. 6, pp. 432-437, 2004.

[16] U. Daniluk, J. Daniluk, M. Krasnodebska, J. M. Lotowska, M. E. Sobaniec-Lotowska, and D. M. Lebensztejn, "The combination of fecal calprotectin with ESR, CRP and albumin discriminates more accurately children with Crohn's disease," Advances in Medical Sciences, vol. 64, no. 1, pp. 9-14, 2019.

[17] E. Fairclough, E. Cairns, J. Hamilton, and C. Kelly, "Evaluation of a modified early warning system for acute medical admissions and comparison with C-reactive protein/albumin ratio as a predictor of patient outcome," Clinical Medicine, vol. 9, no. 1, pp. 30-33, 2009.
[18] J. Wu, W. Tan, L. Chen, Z. Huang, and S. Mai, "Clinicopathologic and prognostic significance of C-reactive protein/albumin ratio in patients with solid tumors: an updated systemic review and meta-analysis," Oncotarget, vol. 9, no. 17, pp. 13934-13947, 2018.

[19] N. Seringec Akkececi, G. Yildirim Cetin, H. Gogebakan, and C. Acipayam, "The C-reactive protein/albumin ratio and complete blood count parameters as indicators of disease activity in patients with Takayasu arteritis," Medical Science Monitor, vol. 25, pp. 1401-1409, 2019.

[20] G. Qin, J. Tu, L. Liu et al., "Serum albumin and C-reactive protein/albumin ratio are useful biomarkers of Crohn's disease activity," Medical Science Monitor, vol. 22, pp. 4393-4400, 2016.

[21] G. D’Haens, W. J. Sandborn, B. G. Feagan et al., “A Review of Activity Indices and Efficacy End Points for Clinical Trials of Medical Therapy in Adults With Ulcerative Colitis," Gastroenterology, vol. 132, no. 2, pp. 763-786, 2007.

[22] K. W. Schroeder, W. J. Tremaine, and D. M. Ilstrup, “Coated oral 5-aminosalicylic acid therapy for mildly to moderately active ulcerative colitis. A randomized study," The New England Journal of Medicine, vol. 317, no. 26, pp. 1625-1629, 1987.

[23] W. R. Best, J. M. Becktel, J. W. Singleton, and F. Kern Jr., "Development of a Crohn's Disease Activity Index: National Cooperative Crohn's Disease Study," Gastroenterology, vol. 70, no. 3, pp. 439-444, 1976.

[24] A. K. Demir, A. Demirtas, S. U. Kaya et al., "The relationship between the neutrophil-lymphocyte ratio and disease activity in patients with ulcerative colitis," The Kaohsiung Journal of Medical Sciences, vol. 31, no. 11, pp. 585-590, 2015.

[25] S. Q. Gao, L. D. Huang, R. J. Dai, D. D. Chen, W. J. Hu, and Y. F. Shan, "Neutrophil-lymphocyte ratio: a controversial marker in predicting Crohn's disease severity," International Journal of Clinical and Experimental Pathology, vol. 8, no. 11, pp. 14779-14785, 2015.

[26] M. Xu, M. Cen, X. Chen, H. Chen, X. Liu, and Q. Cao, “Correlation between serological biomarkers and disease activity in patients with inflammatory bowel disease," BioMed Research International, vol. 2019, Article ID 6517549, 7 pages, 2019.

[27] B. RUOT, F. BÉCHEREAU, G. BAYLE, D. BREUILLÉ, and C. OBLED, "The response of liver albumin synthesis to infection in rats varies with the phase of the inflammatory process," Clinical Science (London, England), vol. 102, no. 1, pp. 107-114, 2002.

[28] O. T. Ranzani, F. G. Zampieri, D. N. Forte, L. C. P. Azevedo, and M. Park, "C-reactive protein/albumin ratio predicts 90-day mortality of septic patients," PLoS One, vol. 8, no. 3, article e59321, 2013. 